\title{
Interaction d'un liquide avec une plaque soumise à une explosion : validation expérimentale d'un modèle dynamique linéaire
}

\author{
André Langleta ${ }^{a}$, Grégory Girault et JÉrôme Renard \\ Laboratoire Énergétique Explosions Structures, 63 avenue du Maréchal de Lattre de Tassigny, 18020 Bourges Cedex, France
}

Reçu le 21 mai 2004, accepté le 17 octobre 2005

\begin{abstract}
Résumé - Ce travail fait suite à l'étude théorique de la réponse d'un système plaque-liquide infini, soumis à un champ de pression uniforme se déplaçant avec une vitesse constante [J. Renard, A. Langlet, O. Pennetier, Response of a large plate-liquid system to a moving pressure step. Transient and stationary aspects, J. Sound and Vibration 265 (2003) 699-724]. Dans la première partie de cet article, après avoir rappelé les équations du problème, on applique la méthode numérique établie dans la référence précédente pour calculer la réponse dynamique couplée au champ de pression créé par une détonation. Dans la deuxième partie, on présente le montage expérimental grâce auquel la mesure des déformations sur une plaque reposant sur de l'eau permet de caractériser la réponse dynamique du système à une détonation. L'objectif principal de cet article est de montrer que la modélisation du couplage fluide-structure qui a été effectuée, décrit convenablement la réponse observée, en particulier les ondes accompagnant le front de chargement.
\end{abstract}

Mots clés : Interaction fluide-structure / détonation / chargement mobile

Abstract - Interaction of a liquid with an explosively loaded plate: experimental validation of a linear dynamical model. This work follows the theoretical study of the response of an infinite plate-liquid system submitted to a uniform pressure field moving at a constant speed [J. Renard, A. Langlet, O. Pennetier, Response of a large plate-liquid system to a moving pressure step. Transient and stationary aspects, J. Sound and Vibration 265 (2003) 699-724]. In the first part of this paper, the governing equations are recalled. Finally, the coupled dynamical response of the plate to the pressure field created by a detonation is obtained, using the numerical method established in the previous reference. In the second part, an experimental set-up is presented which permits to characterise, by means of strains measurements on the plate, the response of an explosively loaded plate. The main objective of this paper is to show that the proposed linear model of the fluid-structure interaction can be applied to describe the observed dynamical response, in particular the waves accompanying the moving load front.

Key words: Fluid-structure interaction / detonation / moving load

\section{Introduction}

Parmi les risques rencontrés dans les activités industrielles, figurent ceux liés aux stockages et à l'emploi de substances explosives. L'évaluation de ces risques, de leurs conséquences sur les populations et l'environnement, se heurte à la variété et à la complexité des situations réelles. Pour permettre une compréhension des phénomènes et de leurs effets, de nombreuses modélisations théoriques ou expérimentales ont été présentées [3,4]. Fréquemment,

a Auteur correspondant :

andre.langlet@bourges.univ-orleans.fr les structures peuvent être en contact avec des liquides. Dans ce cas, la description des phénomènes implique une compréhension de l'interaction fluide-structure, c'est-àdire du couplage entre la dynamique de la structure et celle du liquide.

Cet article se place dans le cadre général de l'étude des problèmes d'interaction fluide-structure.

Plus spécialement, notre objectif est de contribuer à la compréhension de l'interaction lorsque la structure est soumise au champ de pression créé par une détonation, laquelle correspond au mode d'explosion le plus rapide et le plus violent, en terme de surpression. 


\section{Nomenclature}

\begin{tabular}{|c|c|}
\hline$\rho$ & masse volumique de la plaque \\
\hline$h$ & épaisseur de la plaque \\
\hline E & module d'Young de la plaque \\
\hline$v$ & coefficient de Poisson de la plaque \\
\hline$G=E /[2(1+v)]$ & module de cisaillement de la plaque \\
\hline$\rho_{1}$ & masse volumique du liquide \\
\hline$B$ & module de compressibilité du liquide \\
\hline$v_{\mathrm{p}}=\sqrt{E /\left[\rho\left(1-v^{2}\right)\right]}$ & vitesse de propagation des ondes longitudinales de plaque \\
\hline$\kappa$ & facteur de correction de Timoshenko $(\kappa=0,86,[2])$ \\
\hline$v_{\mathrm{s}}=\sqrt{\kappa G / \rho}$ & vitesse modifiée de propagation des ondes de cisaillement \\
\hline$v_{1}=\sqrt{B / \rho_{1}}$ & vitesse de propagation des ondes dans le liquide \\
\hline$r_{0}=h / \sqrt{12}$ & rayon de giration de la plaque \\
\hline$\theta=v_{\mathrm{s}} / v_{\mathrm{p}}$ & vitesse non-dimensionnelle des ondes de cisaillement \\
\hline$\delta=v_{\mathrm{l}} / v_{\mathrm{p}}$ & vitesse non-dimensionnelle des ondes acoustiques dans le liquide \\
\hline$\mu=\rho_{1} /(\rho \sqrt{12})$ & masse volumique non-dimensionnelle du liquide \\
\hline$(R, Z)=(r, z) / r_{0},(X, Z)=(x, z) / r_{0}$ & $\begin{array}{l}\text { variables non-dimensionnelles de repérage dans un système de coordonnées, } \\
\text { respectivement cylindrique et cartésien }\end{array}$ \\
\hline$T=t /\left(r_{0} / v_{\mathrm{p}}\right)$ & variable non-dimensionnelle de temps \\
\hline$\Psi$ & rotation d'une section droite de plaque \\
\hline$W=w / r_{0}$ & déplacement non-dimensionnel hors du plan de la plaque \\
\hline$P_{\mathrm{ext}}=p_{\mathrm{ext}} /\left(\rho v_{\mathrm{p}}^{2} \sqrt{12}\right)$ & pression extérieure non-dimensionnelle \\
\hline$P_{\mathrm{int}}=p_{\mathrm{int}} /\left(\rho v_{\mathrm{p}}^{2} \sqrt{12}\right)$ & pression non-dimensionnelle exercée par le fluide sur la plaque \\
\hline$\Phi=\varphi /\left(r_{0} v_{\mathrm{p}}\right)$ & potentiel non-dimensionnel des vitesses dans le liquide \\
\hline$V=v / v_{\mathrm{p}}$ & vitesse non-dimensionnelle de propagation du front de chargement \\
\hline$Y=X-V T$ & abscisse relative utilisée en analyse stationnaire \\
\hline$\varepsilon=-\sqrt{3} \frac{\partial \Psi}{\partial R}$ & déformation de flexion sur la plaque (pour $z=h / 2$ ) \\
\hline$d_{\mathrm{N}}$ & distance normale entre les centres de détonation et de la plaque impactée \\
\hline$\Delta p^{+}$ & valeur maximale de surpression \\
\hline$t^{+}$ & durée de la phase de surpression \\
\hline$\Delta p^{-}$ & valeur maximale de dépression \\
\hline$t^{-}$ & durée de la phase de dépression \\
\hline$t_{\mathrm{A}}$ & temps d'arrivée de la détonation \\
\hline$\Delta p_{\mathrm{A}}^{+}$ & surpression à l'instant $t_{\mathrm{A}}$ \\
\hline$e_{\mathrm{B}}$ & énergie libérée par la détonation \\
\hline$r_{\mathrm{B}}$ & rayon du confinement \\
\hline
\end{tabular}

Afin d'apporter des conclusions générales nous avons étudié le cas d'une plaque couplée à un liquide et balayée par l'onde de choc créée par une détonation. Le chargement appliqué est un chargement variable, ou mobile, c'est-à-dire un champ de pression se déplaçant avec une certaine vitesse.

Pour prédire la réponse de la plaque aux ondes de chocs, il est intéressant d'étudier le problème unidimensionnel : celui d'une bande soumise à une pression constante se déplaçant à une vitesse constante. Dans l'article [5], ce problème idéalisé est résolu pour les plaques ou les poutres non couplées. Les résultats confirment que les hypothèses de Mindlin-Reissner sont les plus adaptées pour une telle modélisation de la plaque en dynamique rapide. Dans l'article [1] ce problème idéalisé est étendu au couplage fluide-structure en considérant que la plaque est en contact avec un fluide compressible en mouvement irrotationnel à potentiel des vitesses. Lorsque le chargement consiste en une pression constante animée d'une vitesse constante, il est possible d'obtenir des solutions analytiques au problème couplé, pour toutes les valeurs possibles de la vitesse de progression du front de pression. Sous certaines conditions, ces solutions analytiques exactes peuvent servir de guide pour l'interprétation des résultats expérimentaux. Elles ont, en outre, servi de références pour valider un schéma numérique basé sur la méthode des différences finies.

Le chargement de détonation sur la plaque est parfaitement connu [6].

L'application de ce chargement implique, d'une part, la formulation des équations couplées dans un système de coordonnées cylindriques, et d'autre part, le calcul numérique de la réponse avec les coordonnées cylindriques. 
Le but de cet article est de montrer que les résultats théoriques sont, de plus, corroborés par les résultats d'une étude expérimentale originale.

Dans ce qui suit, nous rappellerons d'abord l'essentiel des résultats tant analytiques que numériques. Puis, nous présenterons l'étude expérimentale et ses résultats qui seront confrontés avec ceux de l'étude théorique.

\section{Modélisation}

Pour décrire la réponse de la plaque couplée au liquide, nous nous plaçons dans le cadre de la dynamique linéaire.

Le champ de déplacement de la plaque (Fig. 1) correspond aux hypothèses cinématiques de Mindlin-Reissner, selon lesquelles le mouvement est décrit par : la rotation $\Psi$ de la section droite, le déplacement $W$ perpendiculaire au plan moyen de la plaque. La plaque est soumise à la pression « extérieure » $P_{\text {ext }}$ et à la pression $P_{\text {int }}$ du fluide. Concernant l'établissement des équations dynamiques de la plaque, on peut consulter [2].

Le liquide en contact avec la plaque est supposé compressible et non visqueux; son mouvement est considéré irrotationnel et à potentiel de vitesses $\Phi$.

Le système d'équations couplées est écrit avec des variables non-dimensionnelles (représentées en lettres capitales), en prenant comme référence pour les longueurs $r_{0}=h / \sqrt{12}$ et pour les temps $r_{0} / v_{\mathrm{p}}$, ( $h$ est l'épaisseur de la plaque, $v_{\mathrm{p}}$ désigne la vitesse des ondes longitudinales dans la plaque). De plus, les paramètres sans dimension suivants ont été introduits : $\theta=v_{\mathrm{s}} / v_{\mathrm{p}}, \delta=v_{\mathrm{l}} / v_{\mathrm{p}}$, $\mu=\rho_{\mathrm{l}} /[\rho \sqrt{12}]$, avec $: v_{\mathrm{l}}$ vitesse du son dans le liquide, $v_{\mathrm{s}}$ vitesse corrigée des ondes de cisaillement dans la plaque, $\rho$ et $\rho_{1}$ masses volumiques respectivement de la plaque et $\mathrm{du}$ liquide.

En coordonnées cartésiennes, nous obtenons le système couplé :

$$
\begin{aligned}
\frac{\partial^{2} W}{\partial T^{2}} & =\theta^{2}\left(\frac{\partial^{2} W}{\partial X^{2}}-\frac{\partial \Psi}{\partial X}\right)+\left.\mu \frac{\partial \Phi}{\partial T}\right|_{Z=0}-P_{\mathrm{ext}} \\
\frac{\partial^{2} \Psi}{\partial T^{2}} & =\frac{\partial^{2} \Psi}{\partial X^{2}}+\theta^{2}\left(\frac{\partial W}{\partial X}-\Psi\right) \\
\frac{\partial^{2} \Phi}{\partial T^{2}} & =\delta^{2}\left(\frac{\partial^{2} \Phi}{\partial X^{2}}+\frac{\partial^{2} \Phi}{\partial Z^{2}}\right) \\
\frac{\partial W}{\partial T} & =-\left.\frac{\partial \Phi}{\partial Z}\right|_{Z=0}
\end{aligned}
$$

Les équations (1) et (2) décrivent le mouvement de la plaque sous l'action de la pression extérieure $P_{\text {ext }}$ et de la pression exercée par le liquide : $\mu(\partial \Phi / \partial T)_{z=0}$. L'équation (3) est une équation de Helmoltz décrivant le mouvement du fluide. La continuité des vitesses normales à l'interface entre le fluide et la plaque se traduit par (4).

Pour traiter le cas d'un chargement à symétrie cylindrique, tel que celui de la détonation, on considère

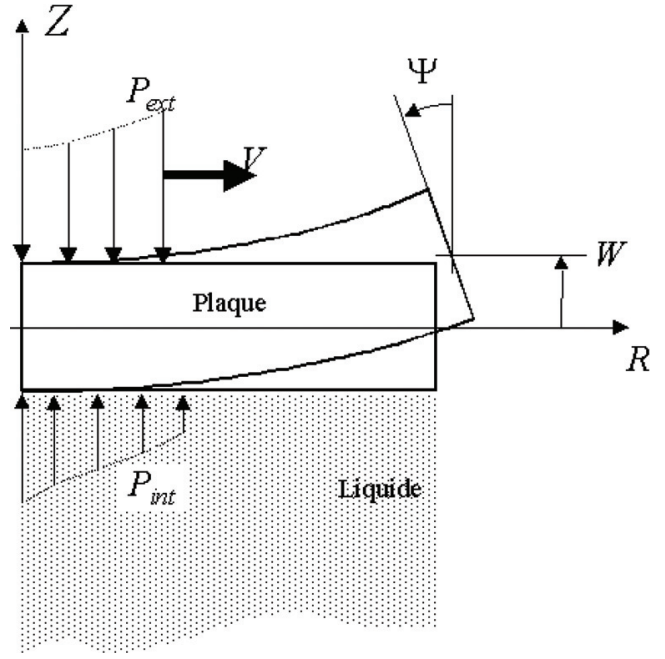

Fig. 1. Cinématique de la plaque et chargement.

maintenant le problème axisymétrique. L'hypothèse d'axisymétrie se traduit par : $W=W(R, T), \Psi=$ $\Psi(R, T), \Phi=\Phi(R, Z, T)$.

En coordonnées cylindriques, et en tenant compte de l'axisymétrie, nous obtenons le système :

$$
\begin{aligned}
\frac{\partial^{2} W}{\partial T^{2}}= & \theta^{2}\left(\frac{\partial^{2} W}{\partial R^{2}}+\frac{1}{R} \frac{\partial W}{\partial R}-\frac{\partial \Psi}{\partial R}-\frac{\Psi}{R}\right) \\
& +\left.\mu \frac{\partial \Phi}{\partial T}\right|_{Z=0}-P_{\text {ext }} \\
\frac{\partial^{2} \Psi}{\partial T^{2}}= & \frac{\partial^{2} \Psi}{\partial R^{2}}+\frac{1}{R} \frac{\partial \Psi}{\partial R}-\frac{\Psi}{R^{2}}+\theta^{2}\left(\frac{\partial W}{\partial R}-\Psi\right) \\
\frac{\partial^{2} \Phi}{\partial T^{2}}= & \delta^{2}\left(\frac{\partial^{2} \Phi}{\partial R^{2}}+\frac{1}{R} \frac{\partial \Phi}{\partial R}+\frac{\partial^{2} \Phi}{\partial Z^{2}}\right) \\
\frac{\partial W}{\partial T}= & -\left.\frac{\partial \Phi}{\partial Z}\right|_{Z=0}
\end{aligned}
$$

\section{Solutions analytiques stationnaires dans le cas unidirectionnel}

Pour décrire le mouvement du système dans un repère lié au front de chargement qui se déplace avec la vitesse constante $V$, on définit la coordonnée : $Y=X-V T$. Les variables ne dépendent alors plus explicitement du temps, mais seulement de $Y$ et $Z: W=W(Y), \Psi=\Psi(Y)$, $\Phi=\Phi(Y, Z)$. Alors, les dérivées partielles deviennent des 
dérivées ordinaires : $\partial / \partial X=\mathrm{d} / \mathrm{d} Y, \partial / \partial T=-V \mathrm{~d} / \mathrm{d} Y$. Nous obtenons :

$$
\begin{aligned}
V^{2} \frac{\mathrm{d}^{2} W}{\mathrm{~d} Y^{2}} & =\theta^{2}\left(\frac{\mathrm{d}^{2} W}{\mathrm{~d} Y^{2}}-\frac{\mathrm{d} \Psi}{\mathrm{d} Y}\right)-\mu V\left(\frac{\partial \Phi}{\partial Y}\right)_{Z=0}-P_{\mathrm{ext}}(Y) \\
V^{2} \frac{\mathrm{d}^{2} \Psi}{\mathrm{d} Y^{2}} & =\frac{\mathrm{d}^{2} \Psi}{\mathrm{d} Y^{2}}+\theta^{2}\left(\frac{\mathrm{d} W}{\mathrm{~d} Y}-\Psi\right) \\
V^{2} \frac{\partial^{2} \Phi}{\partial Y^{2}} & =\delta^{2}\left(\frac{\partial^{2} \Phi}{\partial Y^{2}}+\frac{\partial^{2} \Phi}{\partial Z^{2}}\right) \\
V \frac{\mathrm{d} W}{\mathrm{~d} Y} & =\left(\frac{\partial \Phi}{\partial Z}\right)_{Z=0}
\end{aligned}
$$

Les solutions $W, \Psi, \Phi$, de ce système sont des ondes qui apparaissent figées pour l'observateur lié au front de chargement. Nous les appelons solutions stationnaires. La vitesse de ces ondes est égale à celle du front de chargement.

L'obtention analytique de ces solutions est présentée dans l'article [1] qui traite complètement le cas où $V>$ $\delta$. Le cas où $V<\delta$ est plus difficile à résoudre car un sous-ensemble du système devient elliptique; néanmoins les solutions peuvent encore être obtenues analytiquement par les méthodes de transformées [7].

\section{Approximation des conditions de frontières. Système couplé}

Dans cette section, les dérivées partielles sont exprimées à l'interface plaque liquide ou sur l'axe de symétrie, ce qui conduit à une forme du système couplé adaptée à la résolution numérique.

- Si $R=0$, la condition de symétrie impose :

$$
\left.\frac{\partial W}{\partial R}\right|_{R=0}=\left.\frac{\partial \Phi}{\partial R}\right|_{R=0}=0 \quad \text { et } \quad \Psi=0
$$

- Si $R \rightarrow 0$,

$$
\lim _{R \rightarrow 0}=\frac{\Psi}{R}=\left.\frac{\partial \Psi}{\partial R}\right|_{R=0} \quad \text { et } \quad \lim _{R \rightarrow 0} \frac{\partial \Psi}{\partial R}=\left.\frac{\partial^{2} \Psi}{\partial R^{2}}\right|_{R=0}
$$

- Si $Z=0$, un développement de Taylor à l'ordre 2 conduit à :

$$
\begin{aligned}
\left.\frac{\partial^{2} \Phi}{\partial Z^{2}}\right|_{Z=0} & =\frac{2}{\Delta Z^{2}}\left[\Phi(\Delta Z)-\Phi(0)-\left.\Delta Z \frac{\partial \Phi}{\partial Z}\right|_{Z=0}\right] \\
& =\frac{2}{\Delta Z^{2}}\left[\Phi(\Delta Z)-\Phi(0)+\Delta Z \frac{\partial W}{\partial T}\right]
\end{aligned}
$$

Si les termes contenant les dérivées temporelles sont regroupées dans les membres de gauche, et les termes contenant les dérivées en espace sont regroupés dans les membres de droite, on obtient :

$$
\begin{aligned}
& R=0, Z=0 \\
& \frac{\partial^{2} W}{\partial T^{2}}-\left.\mu \frac{\partial \Phi}{\partial T}\right|_{Z=0}=2 \theta^{2}\left[\frac{\partial^{2} W}{\partial R^{2}}+\frac{\partial \Psi}{\partial R}\right]-P_{\text {ext }}(R, T) \\
& \Psi(0)=0 \\
& \frac{\partial^{2} \Phi}{\partial T^{2}}-\frac{2 \delta^{2}}{\Delta Z} \frac{\partial W}{\partial T}=2 \delta^{2}\left\{\frac{\partial^{2} \Phi}{\partial R^{2}}+\frac{1}{\Delta Z^{2}}[\Phi(\Delta Z)-\Phi(0)]\right\}
\end{aligned}
$$

$R>0, Z=0$

$$
\begin{aligned}
& \frac{\partial^{2} W}{\partial T^{2}}-\left.\mu \frac{\partial \Phi}{\partial T}\right|_{Z=0}= \\
& \theta^{2}\left[\frac{\partial^{2} W}{\partial R^{2}}+\frac{1}{R} \frac{\partial W}{\partial R}-\frac{\partial \Psi}{\partial R}-\frac{\Psi}{R}\right]-P_{\text {ext }}(R, T) \\
& \frac{\partial^{2} \Psi}{\partial T^{2}}=\frac{\partial^{2} \Psi}{\partial R^{2}}+\frac{1}{R} \frac{\partial \Psi}{\partial R}-\frac{\Psi}{R^{2}}+\theta^{2}\left[\frac{\partial W}{\partial R}-\Psi\right] \\
& \frac{\partial^{2} \Phi}{\partial T^{2}}-\frac{2 \delta^{2}}{\Delta Z} \frac{\partial W}{\partial T}= \\
& \delta^{2}\left\{\frac{\partial^{2} \Phi}{\partial R^{2}}+\frac{1}{R} \frac{\partial \Phi}{\partial R}+\frac{2}{\Delta Z^{2}}[\Phi(\Delta Z)-\Phi(0)]\right\}
\end{aligned}
$$

$R=0, Z<0$

$$
\frac{\partial^{2} \Phi}{\partial T^{2}}=\delta^{2}\left[2 \frac{\partial^{2} \Phi}{\partial R^{2}}+\frac{\partial \Phi}{\partial Z^{2}}\right]
$$

$R>0, Z<0$

$$
\frac{\partial^{2} \Phi}{\partial T^{2}}=\delta^{2}\left[\frac{\partial^{2} \Phi}{\partial R^{2}}+\frac{1}{R} \frac{\partial \Phi}{\partial R}+\frac{\partial \Phi}{\partial Z^{2}}\right]
$$

\section{Discrétisation du système par des différences finies}

Les dérivées d'ordre 1 et 2 sont exprimées en des points discrétisés du domaine spatial : $(R, Z) \equiv\left(i_{R} \Delta R, i_{Z} \Delta Z\right)$, $i_{R}=1, \ldots, N_{R}, i_{Z}=1, \ldots, N_{Z}$, et du domaine temporel : $T=k \Delta T$. On applique les formules classiques des différences finies centrées :

$$
\begin{aligned}
& \frac{\partial F}{\partial R}=\frac{F\left(i_{R}+1, i_{Z}\right)-F\left(i_{R}-1, i_{Z}\right)}{2 \Delta R} \\
& \frac{\partial^{2} F}{\partial R^{2}}=\frac{F\left(i_{R}+1, i_{Z}\right)-2 F\left(i_{R}, i_{Z}\right)+F\left(i_{R}-1, i_{Z}\right)}{\Delta R^{2}} \\
& \frac{\partial F}{\partial T}=\frac{F_{\mathrm{s}}-F_{\mathrm{p}}}{2 \Delta T}, \quad \frac{\partial^{2} F}{\partial T^{2}}=\frac{F_{\mathrm{s}}-2 F+F_{\mathrm{p}}}{\Delta T^{2}}
\end{aligned}
$$

où : $F\left(i_{R}, i_{Z}\right)=F$ désigne la valeur d'une fonction $F$ au point $\left(i_{R}, i_{Z}\right)$ à l'instant actuel $k ; F_{\mathrm{p}}\left(i_{R}, i_{Z}\right)=F_{\mathrm{p}}$ et 
$F_{\mathrm{s}}\left(i_{R}, i_{Z}\right)=F_{\mathrm{s}}$, désignent respectivement les valeurs de la fonction $F$ au point $\left(i_{R}, i_{Z}\right)$ à l'instant précédent $k-1$ et à l'instant suivant $k+1$.

Après avoir remplacé les dérivées partielles par leurs approximations respectives en différences finies, et effectué les regroupements nécessaires, on obtient les valeurs des fonctions à l'instant « suivant» au point $M\left(i_{R}, i_{Z}\right)$, connaissant la valeur actuelle et la valeur précédente de la fonction considérée. On obtient le schéma explicite d'ordre deux :

$$
\begin{gathered}
\left\{\begin{array}{l}
W_{\mathrm{s}} \\
\Phi_{\mathrm{s}}
\end{array}\right\}=\frac{2}{1+\alpha \beta}\left[\begin{array}{cc}
1 & \alpha \\
-\beta & 1
\end{array}\right]\left\{\begin{array}{l}
W \\
\Phi
\end{array}\right\} \\
+\frac{1}{1+\alpha \beta}\left[\begin{array}{cc}
\alpha \beta-1 & -2 \alpha \\
2 \beta & \alpha \beta-1
\end{array}\right]\left\{\begin{array}{l}
W_{\mathrm{p}} \\
\Phi_{\mathrm{p}}
\end{array}\right\} \\
+\frac{\Delta T^{2}}{1+\alpha \beta}\left[\begin{array}{cc}
1 & \alpha \\
-\beta & 1
\end{array}\right]\left\{\begin{array}{l}
Q_{1} \\
Q_{2}
\end{array}\right\} \\
\Psi_{\mathrm{s}}=2 \Psi-\Psi_{\mathrm{p}}+\Delta T^{2} C
\end{gathered}
$$

Avec :

$$
\begin{aligned}
& \alpha=\frac{1}{2} \mu \Delta T \quad \beta=-\delta^{2} \frac{\Delta T}{\Delta Z} \\
& C=\frac{1}{\Delta R^{2}}\left[\Psi\left(i_{R}+1\right)-2 \Psi\left(i_{R}\right)+\Psi\left(i_{R}-1\right)\right] \\
& +\frac{1}{2 \Delta R^{2}}\left[\frac{\Psi\left(i_{R}+1\right)-\Psi\left(i_{R}-1\right)}{i_{R}}\right]-\frac{1}{\Delta R^{2}} \frac{\Psi\left(i_{R}\right)}{i_{R}^{2}} \\
& -\theta^{2} \Psi\left(i_{R}\right)-\frac{\theta^{2}}{2 \Delta R}\left[W\left(i_{R}+1\right)-W\left(i_{R}-1\right)\right] \\
& i_{R}>0 \Rightarrow Q_{1}=\left[\frac{W\left(i_{R}+1\right)-2 W\left(i_{R}\right)+W\left(i_{R}-1\right)}{\Delta R^{2}}\right. \\
& +\frac{1}{i_{R} \Delta R} \frac{W\left(i_{R}+1\right)-W\left(i_{R}-1\right)}{2 \Delta R} \\
& \left.-\frac{\Psi\left(i_{R}+1\right)-\Psi\left(i_{R}-1\right)}{2 \Delta R}-\frac{\Psi}{i_{R} \Delta R}\right]-P_{\text {ext }}\left(i_{R}\right) \\
& i_{R}=0 \Rightarrow Q_{1}=\frac{2 \theta^{2}}{\Delta R}[W(1)-2 W(0)+W(-1)] \\
& +\frac{\theta^{2}}{\Delta R}[\Psi(1)-\Psi(-1)]-P_{\text {ext }}(0) \\
& i_{R}>0 \Rightarrow Q_{2}=\frac{\delta^{2}}{\Delta R^{2}}\left[\Phi\left(0, i_{R}+1\right)-2 \Phi\left(0, i_{R}\right)\right. \\
& \left.+\Phi\left(0, i_{R}-1\right)\right]+\frac{\delta^{2}}{2 \Delta R^{2}}\left[\frac{\Phi\left(0, i_{R}+1\right)-\Phi\left(0, i_{R}-1\right)}{i_{R}}\right] \\
& +\frac{2 \delta^{2}}{\Delta Z^{2}}\left[\Phi\left(1, i_{R}\right)-\Phi\left(0, i_{R}\right)\right]
\end{aligned}
$$

Tableau 1. Fréquences et longueurs d'ondes des vibrations pour deux valeurs de la vitesse $V$ du front de chargement.

\begin{tabular}{ccc}
\hline$V$ (sans dimension) & 0,25 & 1,2 \\
\hline$v\left(\mathrm{~m} . \mathrm{s}^{-1}\right)$ & 1353 & 6494 \\
\hline$f(\mathrm{kHz})$ & 597 & $6,7 \times 10^{3}$ \\
\hline$\lambda(\mathrm{mm})$ & 2,27 & 0,97 \\
\hline
\end{tabular}

$$
\begin{aligned}
i_{R}=0 \Rightarrow Q_{2}=\frac{2 \delta^{2}}{\Delta R^{2}}[ & \Phi(0,1)-2 \Phi(0,0)+\Phi(0,-1)] \\
+ & \frac{2 \delta^{2}}{\Delta Z^{2}}[\Phi(1,0)-\Phi(0,0)]
\end{aligned}
$$

\section{Solutions numériques explicites : réponse à une explosion}

Le système couplé est hyperbolique et linéaire. Sa résolution est effectuée à partir des équations (24), (25). Pour chaque temps, on calcule les solutions dans l'ensemble du domaine plaque-liquide avec des pas $\Delta R>0$ et $\Delta Z=-\Delta R$.

Le calcul progresse dans le temps avec un pas $\Delta T$. Une condition nécessaire pour que la stabilité numérique soit assurée est que $\Delta T$ soit inférieur au minimum de la condition CFL (Courant-Freidrichs-Lewy) respectivement pour la plaque seule, et pour le liquide seul, $[1,5]$. On sait également que les conditions d'interface imposées par le couplage fluide-structure peuvent elles-même générer des instabilités numériques. Pour éviter d'introduire des instabilités numériques, les conditions d'interface ont été traitées à l'ordre deux au moins, comme pour les domaines liquide ou de la plaque. Une étude théorique approfondie de l'influence des conditions d'interface sur la stabilité numérique serait nécessaire, mais elle dépasserait le cadre de cet article. On peut noter, cependant, que l'apparition des instabilités se traduit par une divergence des solutions qui ne laisse, dans ce cas, aucun doute sur l'invalidité des résultats.

Enfin, pour que la précision des résultats soit bonne, on admet que le pas d'espace doit être au plus égal au dixième de la longueur d'onde des oscillations que l'on veut décrire. Pour une vitesse $V$ donnée du front de chargement, la fréquence et la longueur d'onde déduites de l'analyse stationnaire peuvent servir de références. Sur une plaque d'aluminium d'épaisseur $h=0,5 \mathrm{~mm}$ $\left(r_{0}=0,144 \mathrm{~mm}\right)$ couplée avec de l'eau, les fréquences et les longueurs d'ondes $\lambda$ sont les suivantes (Tab. 1).

Le tableau 2 fournit les caractéristiques matérielles de la plaque et du liquide utilisées pour ces calculs.

Les résultats numériques présentés dans cet article correspondent à des pas d'espace $\Delta R=0,2 h / r_{0}$ et des pas de temps $\Delta T=\Delta R / 8500$ qui permettent de s'assurer de la stabilité numérique des résultats. Ainsi, le calcul explicite est bien adapté à la description de la réponse dynamique. Son inconvénient est de nécessiter des durées assez importantes, compte tenu des pas utilisés. En contrepartie, chaque étape du calcul est relativement simple et requiert un volume de mémoire raisonnable (par exemple : $30 \mathrm{Mo}$, pour une plaque de $35 \mathrm{~cm}$ de rayon, 
Tableau 2. Caractéristiques de la plaque et du liquide.

\begin{tabular}{cccccc}
\hline & $\theta$ & $v_{\mathrm{p}}$ & $E$ & $\nu$ & $\rho$ \\
\hline Plaque & 0,55 & $5412 \mathrm{~m} \cdot \mathrm{s}^{-1}$ & $72 \mathrm{GPa}$ & 0,3 & $2790 \mathrm{~kg} \cdot \mathrm{m}^{-3}$ \\
\hline
\end{tabular}

\begin{tabular}{cccc}
\hline & $\delta$ & $v_{1}$ & $\rho_{1}$ \\
\hline Liquide & 0,28 & $1500 \mathrm{~m} \cdot \mathrm{s}^{-1}$ & $1000 \mathrm{~kg} \cdot \mathrm{m}^{-3}$ \\
\hline
\end{tabular}

avec : $\Delta r=0,2 \mathrm{~mm}, \Delta t=2,94 \times 10^{-8} \mathrm{~s}, N_{R}=1400$, $\left.N_{Z}=924\right)$.

La pression $P_{\text {ext }}$ représentant le chargement extérieur est calculée à chaque instant $T$. Dans le cas de la détonation, on se réfère aux formules analytiques antérieures [6].

\section{Résultats numériques}

Le système est initialement au repos. Dans le liquide les vitesses des ondes sont inférieures aux vitesses des ondes dans la plaque. Comme on s'intéresse à la solution numérique avant la première réflexion d'une onde sur un bord, la profondeur du fluide peut être choisie inférieure au rayon de la plaque (ce qui diminue la taille mémoire dans les calculs). Dans ce cas, les ondes observées sont comparables à celles qui se développent dans un système plaque-liquide infini, sans influence des bords.

Dans la figure 2, on a représenté, pendant une durée de $350 \mu \mathrm{s}$ : l'évolution $P_{\text {ext }}(R, T)$ du chargement, les déplacements $W(R, T)$, et les déformations radiales calculées sur la plaque, soit : $\varepsilon=-\sqrt{3} \partial \Psi / \partial R$. Le temps zéro correspond au début d'application de la pression au centre $r=0$ de la plaque.

On peut ainsi appréhender les caractéristiques typiques de la réponse dynamique de la plaque à un chargement mobile.

Le calcul est effectué pour des plaques en aluminium (de 0,5 mm et $5 \mathrm{~mm}$ d'épaisseur, dont les caractéristiques sont données dans le Tab. 2) reposant sur de l'eau. Les plaques sont soumises à la détonation dans l'atmosphère d'une charge équivalente à environ $0,3 \mathrm{~g}$ de TNT, placée à une distance $d_{\mathrm{N}}=0,283 \mathrm{~m}$ au-dessus du centre de chaque plaque.

La figure 2a montre l'évolution spatio-temporelle de la pression de détonation. Les tracés sont effectués toutes les $35 \mu$ s. La pression nulle correspond à la pression atmosphérique.

Le front de chargement progresse avec une vitesse décroissante en suivant la ligne en pointillés de la figure $2 \mathrm{~b}$.

Les ondes se développent à partir du point d'application de la pression extérieure. Au cours du temps, ces ondes envahissent progressivement la plaque.

Lorsque l'on s'éloigne vers l'avant du front, le mouvement de la plaque paraît s'organiser pour former des vibrations, figure $2 \mathrm{~b}$ et surtout, figures $2 \mathrm{c}, \mathrm{d}$.

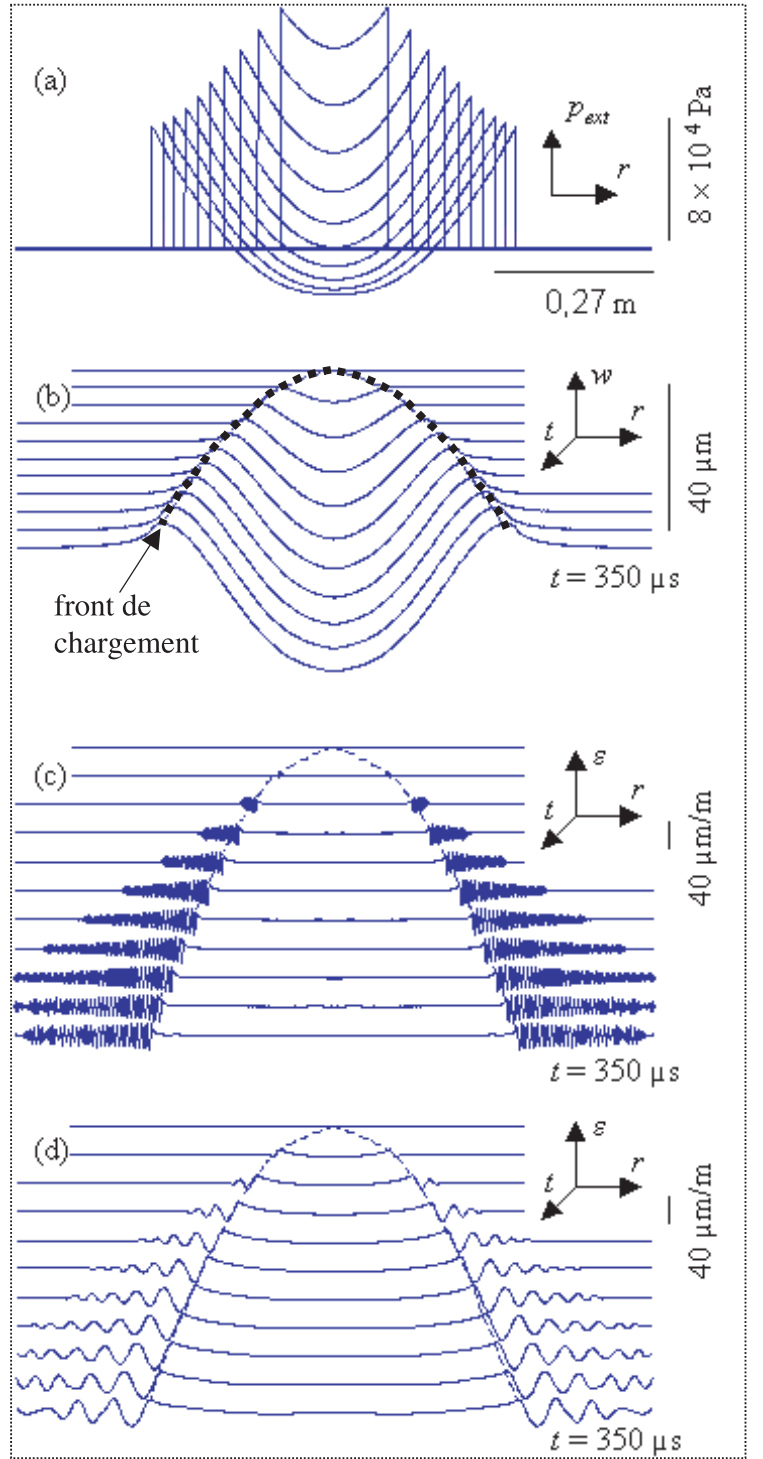

Fig. 2. (a) Chargement sur la plaque créé par une détonation $\left(d_{\mathrm{N}}=0,283 \mathrm{~m}\right.$ et $\left.r_{\mathrm{B}}=0,05 \mathrm{~m}\right)$. (b) Ondes de déplacements sur la plaque de $0,5 \mathrm{~mm}$ d'épaisseur. (c) Ondes de déformations sur la plaque de $0,5 \mathrm{~mm}$ d'épaisseur. (d) Ondes de déformations sur la plaque de $5 \mathrm{~mm}$ d'épaisseur.

Si le chargement était une pression constante se déplaçant avec la vitesse constante $V$, la fréquence et la longueur d'onde des vibrations en avant du front tendraient exactement vers celles prévues par la théorie stationnaire [1]. Dans le cas présent, il s'agit d'une superposition d'ondes sur la plaque, générées par le chargement non-uniforme de la détonation.

Lorsque l'on s'éloigne vers l'arrière du front, le déplacement $W$ est dominé par des oscillations dont l'amplitude et la longueur d'onde ne cessent d'évoluer avec le temps et l'espace. Cette partie dite « transitoire» du mouvement est toujours présente dans la réponse de la plaque couplée. Elle ne peut être obtenue par l'analyse stationnaire mais seulement, dans notre étude, par la résolution numérique. 


\section{3 Étude expérimentale}

\subsection{La détonation}

La détonation est un phénomène déterministe et parfaitement reproductible. La courbe de pression réfléchie en un point peut se résumer à une montée brutale en pression (durée de l'ordre de trois microsecondes) suivie d'une décroissance rapide, figure 3 . On observe une phase de surpression, d'intensité maximale $\Delta p^{+}$, de durée $t^{+}$, suivie par une phase de dépression d'intensité maximale $\Delta p^{-}$bornée physiquement par la pression absolue nulle.

Le milieu réactif utilisé dans notre étude est un mélange de propane et d'oxygène en proportions stœchiométriques. Son énergie spécifique est 12,14 MJ.m ${ }^{-3}$. Pour préserver les concentrations initiales, un confinement est nécessaire. La technique retenue est de former une bulle de savon en injectant le mélange gazeux dans une solution aqueuse d'oléate de sodium.

Deux électrodes en tungstène, sur lesquelles est soudé un filament de cuivre, sont situées à l'intérieur de la bulle et à la verticale du centre $\mathrm{O}$ de la plaque.

L'amorçage de la détonation est créé dans la bulle par la vaporisation du filament sous l'effet d'un courant de forte intensité. La synchronisation (temps $t=0$ ) des mesures dynamiques est donnée par l'explosion du filament.

Dans le mélange réactif confiné, l'onde de choc se propage à la vitesse dite de Chapman-Jouguet (par exemple 1500 à $3000 \mathrm{~m} . \mathrm{s}^{-1}$ ). Dans l'atmosphère, l'onde de choc transmise est sphérique. Elle atteint le point $\mathrm{O}$ au « temps d'arrivée $» t_{\mathrm{A}}$; la surpression à cet instant est notée $\Delta p_{\mathrm{A}}^{+}$. Connaissant $d_{\mathrm{N}}$ et $\Delta p_{\mathrm{A}}^{+}$, par une mesure de pression, on peut connaître l'énergie $e_{\mathrm{B}}$ dégagée par la détonation. À cette valeur $e_{\mathrm{B}}$ correspond un volume de mélange que l'on obtient par les relations énergétiques. On en déduit la valeur $r_{\mathrm{B}} \mathrm{du}$ rayon de la bulle, lequel caractérise la charge explosive.

Les mesures fournies par six capteurs de pression, placés sur des cercles concentriques sont représentées figure 4 afin de bien montrer la symétrie du chargement sur la plaque. Les mesures sont indépendantes de l'angle polaire $\alpha$ mais ne dépendent que du rayon.

Les études expérimentales antérieures menées au laboratoire [6] montrent que, pour un mélange donné, la connaissance de $d_{\mathrm{N}}$ et de $r_{\mathrm{B}}$ permet de calculer analytiquement la répartition spatio-temporelle de la pression réfléchie, $p_{\text {ext }}$, sur la plaque, d'où l'introduction de cette pression dans les codes numériques, comme dans la figure $2 \mathrm{a}$.

\subsection{Dispositif expérimental}

On utilise une cuve de $80 \mathrm{~cm}$ de profondeur, remplie d'eau, à la surface de laquelle sont disposées les plaques étudiées, figure 5. Les plaques sont carrées ( $80 \mathrm{~cm}$ de côté) et sont fixées par une colle au silicone dans un cadre en acier. Ce cadre est maintenu au dessus de la cuve grâce à des supports vissés permettant de s'assurer du contact total de la face inférieure de chaque plaque avec l'eau.

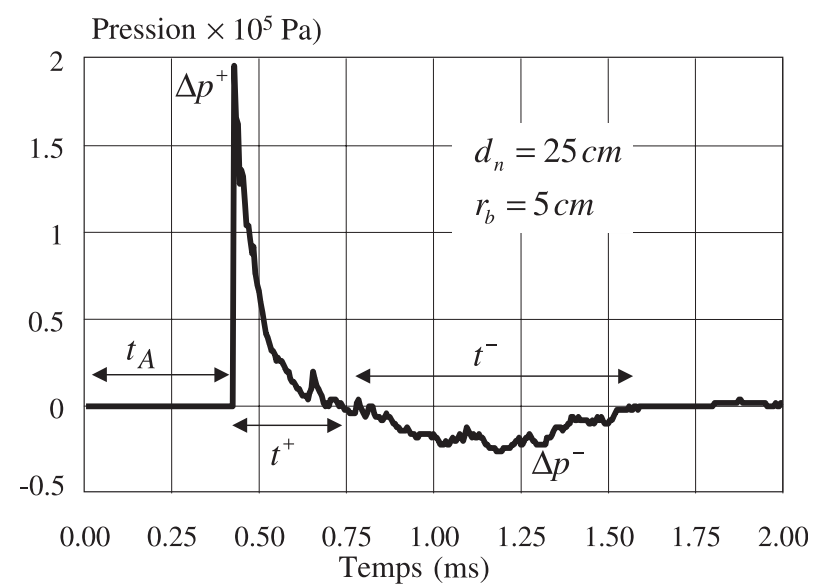

Fig. 3. Profil expérimental type d'une détonation.

Un capteur de pression KISTLER $603 \mathrm{~B}$, placé à une distance $d_{\mathrm{N}}$ du centre de la bulle et à $45^{\circ}$ par rapport à la plaque, permet de connaître $t_{\mathrm{A}}$ et $\Delta p_{\mathrm{A}}^{+}$.

On mesure les déformations de flexion sur la face supérieure de la plaque grâce à des jauges d'extensométrie. La direction de mesure de chaque jauge est radiale, telle que OJ sur la figure 4. La position de chaque jauge est définie par son rayon $r$ ou OJ. On utilise des jauges d'extensométrie VISHAY de 120 ou $350 \Omega$ de résistances nominales. La longueur de grille de ces jauges vaut $0,8 \times 10^{-3} \mathrm{~m}$, ce qui autoriserait des mesures de déformations jusqu'à des fréquences de $6,75 \times 10^{5} \mathrm{~Hz}$. Toutefois, la bande passante $\left[1,6-10^{5}\right] \mathrm{Hz}$ des conditionneurs SEDEME employés limite en pratique l'étude. Il est à noter qu'au-delà de $10^{5} \mathrm{~Hz}$, un étalonnage dynamique de la chaîne de mesure a permis de déterminer l'atténuation et le déphasage subis par les signaux. Ainsi, des déformations de fréquence allant jusqu'à $2 \times 10^{5} \mathrm{~Hz}$ peuvent être étudiées. Enfin l'acquisition des signaux s'effectue sur des oscilloscopes numériques TEKTRONIK à une fréquence d'échantillonnage maximale de $1 \mathrm{GHz}$. Le codage de l'amplitude se fait sur 9 bit.

\section{Résultats et discussion}

Les résultats expérimentaux présentés sont relatifs à deux plaques d'aluminium d'épaisseur différente : plaque $1, h=0,5 \mathrm{~mm}$; plaque $2, h=5 \mathrm{~mm}$. Les caractéristiques mécaniques des plaques et du liquide sont données dans le tableau 2. Les conditions de chargement $\left(d_{\mathrm{N}}=0,283 \mathrm{~m}, r_{\mathrm{B}}=5 \mathrm{~cm}\right)$ et les positions des jauges de déformations $(r=0,28 \mathrm{~m})$ sont identiques. La charge explosive utilisée au laboratoire correspond à environ 0,3 gramme de TNT. En associant la similitude mécanique et la similitude de Hopkinson, il est possible de prévoir les réponses de configurations expérimentales similaires, ou «homothétiques ». Ainsi, si le rapport d'échelle sur les longueurs est $\beta=21,54$, l'explosion d'une sphère gazeuse $^{1}$ de rayon $5 \beta \mathrm{cm}$ (d'équivalent énergétique

\footnotetext{
1 Mélange stœchiométrique propane et oxygène.
} 

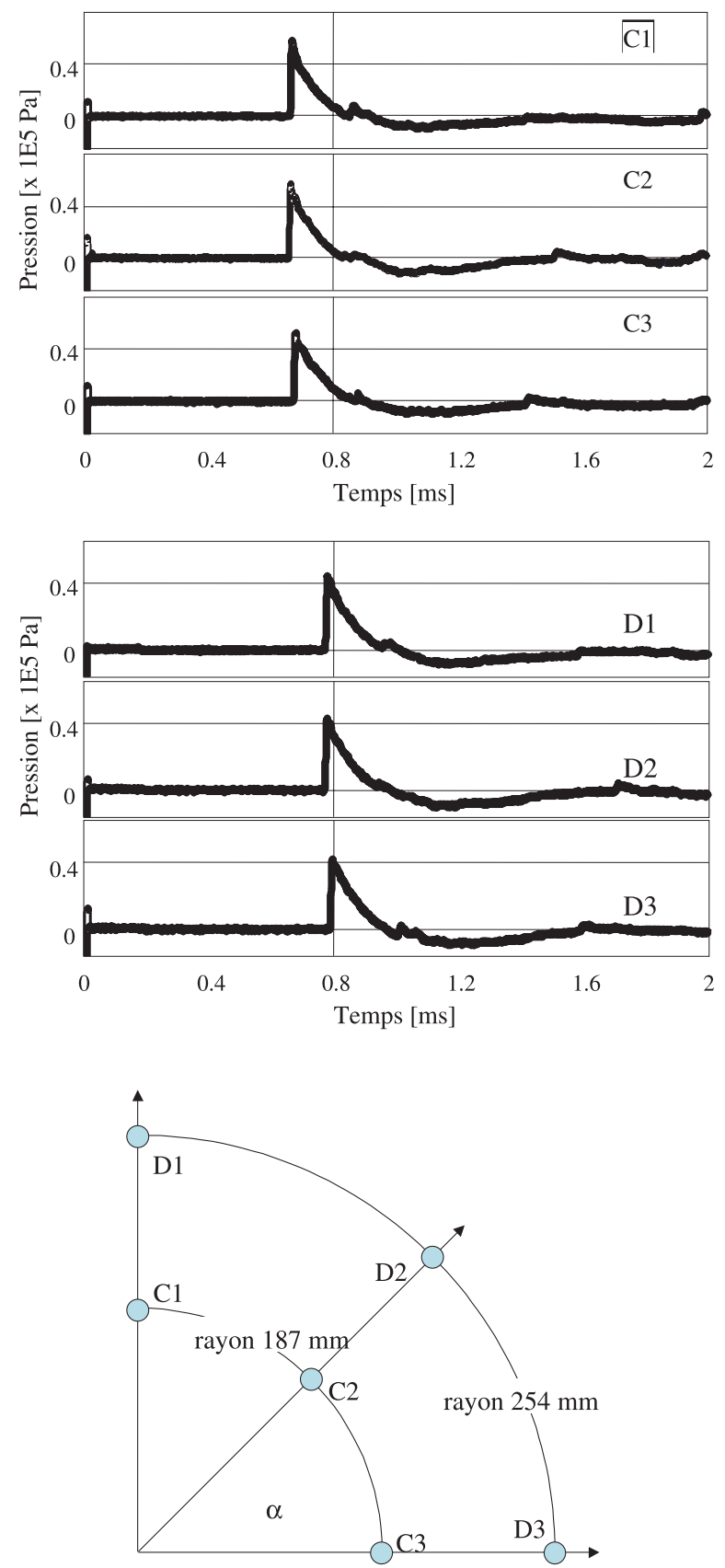

Fig. 4. Répartition spatio-temporelle de la pression sur une plaque $\left(r_{\mathrm{B}}=3,5 \mathrm{~cm}, d_{\mathrm{N}}=28 \mathrm{~cm}\right)$.

$0,3 \times 10^{-3} \beta^{3}=3 \mathrm{~kg}$ de TNT), distante de $0,283 \beta \mathrm{m}$ d'une plaque d'épaisseur $0,05 \beta=1,08 \mathrm{~cm}$, produira sur cette plaque des ondes de déplacements dont les amplitudes sont multipliées par $\beta$ et les fréquences divisées par $\beta$. De plus, en des points homologues, les pressions et les déformations sur la plaque de 0,5 mm d'épaisseur et sur la plaque de 1,08 cm d'épaisseur, auront des amplitudes identiques.

Afin de déterminer le temps d'observation tel que les signaux mesurés ne comportent aucune onde réfléchie par les bords, une jauge de déformations, disposée au rayon maximal de la plaque, détecte la première réflexion. Ainsi
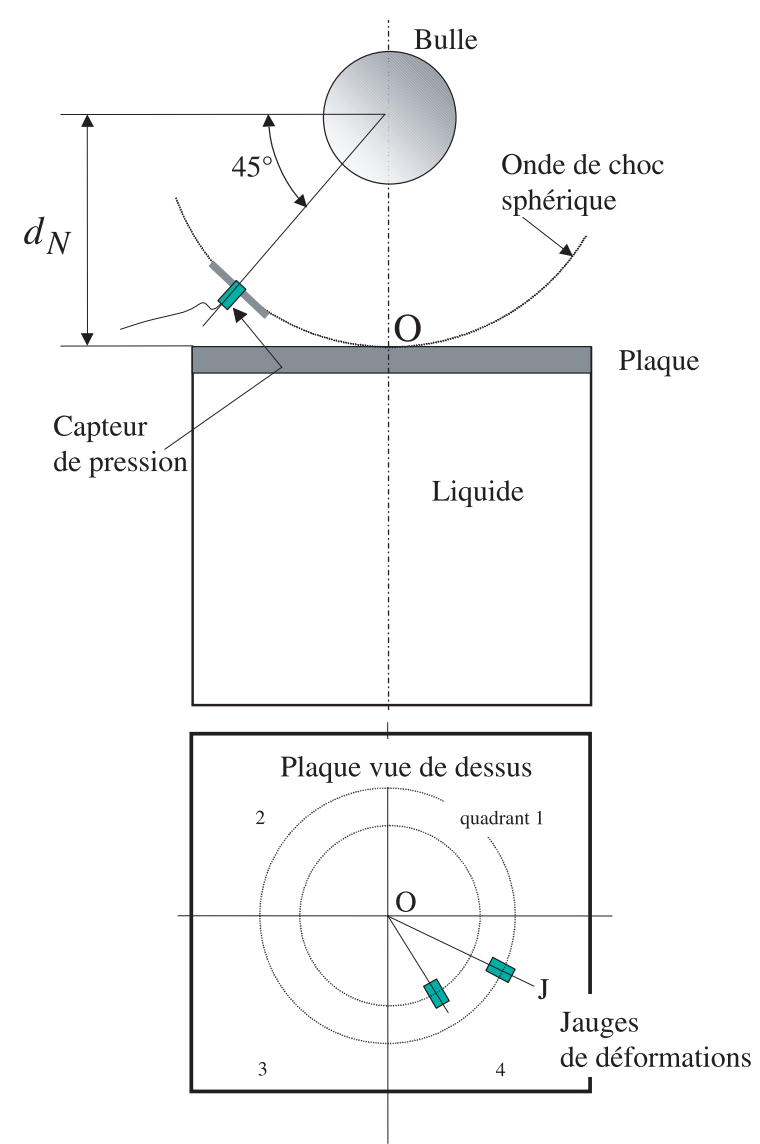

Fig. 5. Schéma du dispositif expérimental.

peut-on distinguer dans les enregistrements, la partie influencée par les réflexions de la partie comparable à une propagation en milieu infini. Dans notre montage, la partie du signal en arrière du front est influencée par les réflexions et ne sera pas exploitée.

D'après les solutions numériques, figure 2, on peut distinguer la zone située en arrière et en avant du front de chargement. L'étude théorique [1] a montré que la fréquence des ondes de la partie en avant du front décroît lorsque l'épaisseur de la plaque augmente. Pour une charge explosive identique $\left(d_{\mathrm{N}}, r_{\mathrm{B}}\right.$ constants $)$, les mouvements possèdent donc des longueurs d'onde d'autant plus faibles et des fréquences d'autant plus grandes que l'épaisseur $h$ est faible, ceci est illustré par le calcul dans les figures 2c,d, et par l'expérience, dans les figures 6 et 7 .

Les signaux expérimentaux de la plaque 2 , figure 7 , se placent dans la bande passante $\left[1,6-10^{5}\right] \mathrm{Hz}$. Par contre, sur la plaque 1 , figure 6 , la fréquence des ondes peut atteindre environ $190 \mathrm{kHz}$ vers $600 \mu$ s et environ $80 \mathrm{kHz}$ vers $725 \mu \mathrm{s}$. On se base alors sur une fréquence moyenne de $135 \mathrm{kHz}$ pour tenir compte de l'atténuation et du déphasage introduits par la chaîne de mesure. On comprend donc que l'amplitude des ondes possédant les plus hautes fréquences soit légèrement sous-estimée.

En ce qui concerne la reproductibilité des mesures, nous bénéficions du caractère déterministe de la réponse étudiée. De plus, la détonation est elle-même 


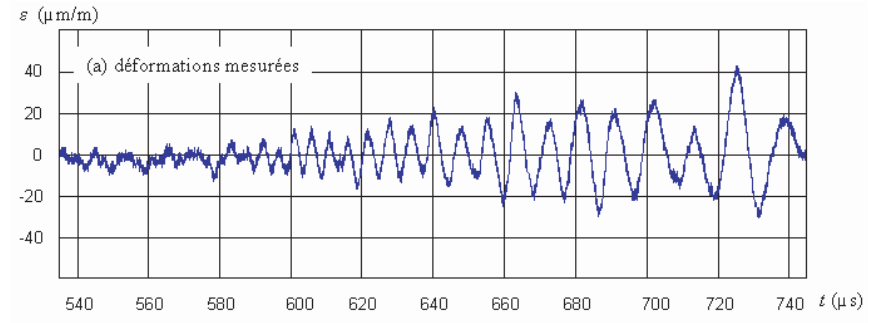

$\varepsilon(\mu \mathrm{m} / \mathrm{m})$

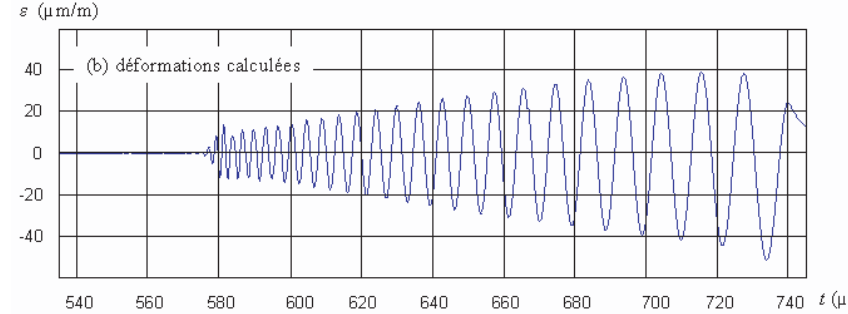

Fig. 6. Déformations sur la plaque 1 à $0,280 \mathrm{~m}$ du centre $\left(d_{\mathrm{N}}=0,283 \mathrm{~m} ; r_{\mathrm{B}}=0,05 \mathrm{~m}\right)$. Comparaison entre l'expérience (a) et le calcul (b).
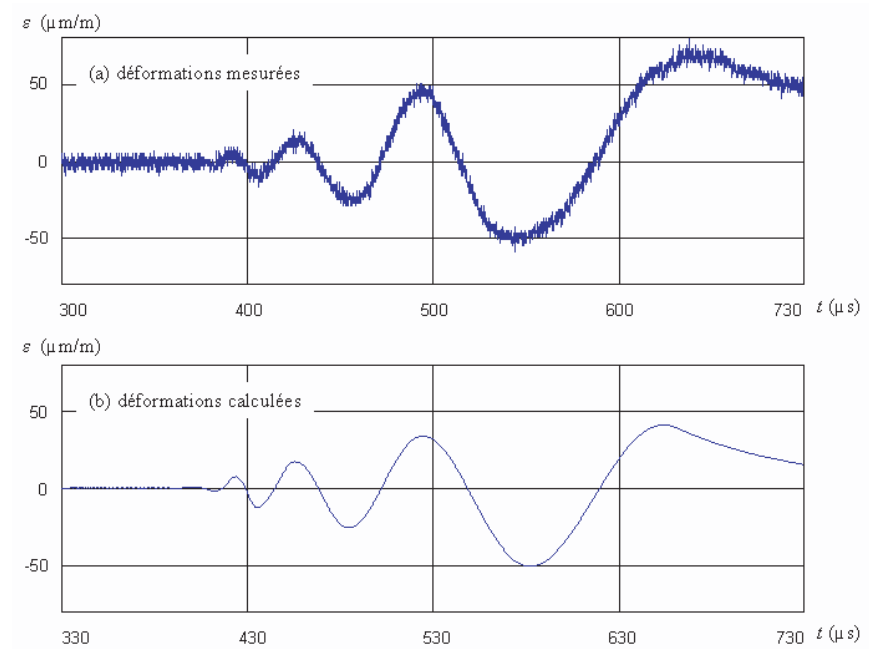

Fig. 7. Déformations sur la plaque 2 à $0,280 \mathrm{~m}$ du centre $\left(d_{\mathrm{N}}=0,283 \mathrm{~m} ; r_{\mathrm{B}}=0,05 \mathrm{~m}\right)$. Comparaison entre l'expérience (a) et le calcul (b).

complètement déterministe. Si les caractéristiques énergétiques de la charge ne varient pas, on observera une réponse mécanique identique. En pratique, la mesure de la pression par le capteur témoin (Fig. 5) permet de vérifier si la détonation produite est conforme aux données de références [6], compte tenu des paramètres expérimentaux actuels : $r_{\mathrm{B}}, d_{\mathrm{N}}$ et mélange stœechiométrique propane et oxygène. Comme ce capteur témoin perturbe le champ de pression, la réponse de la plaque est étudiée sur un quadrant seulement (Fig. 5).

Des erreurs de mesures importantes peuvent être dues aux problèmes de compatibilité électromagnétique avec le dispositif d'amorçage de la détonation. Ces erreurs sont liées aux courants induits dans les câbles et les capteurs placés dans le champ électromagnétique. En pratique on s'aperçoit que ces signaux erronés de déformations sont complètement (eux aussi) déterministes (en fréquence et en amplitude), mais surtout qu'ils peuvent être éliminés par des blindages en aluminium connectés à la même masse électrique.

Le décalage temporel qui subsiste entre les signaux expérimentaux et théoriques (Fig. 7) peut s'expliquer par l'incertitude qui demeure sur le chargement : sphéricité de l'onde de choc, localisation du premier point d'impact, en particulier.

Au terme de notre étude, les résultats expérimentaux sont validés.

On peut en déduire que le modèle théorique permet de décrire correctement les ondes qui accompagnent le front de chargement sur la plaque.

\section{Conclusion}

Dans cette étude, on a considéré la dynamique d'une plaque couplée à un liquide et sollicitée par le champ de pression d'une détonation.

La vitesse de propagation du front de pression est d'abord supersonique, puis décroît vers des valeurs subsoniques. Un tel chargement variable génère dans la plaque et le liquide une superposition d'ondes élastiques. La réponse observée comprend une partie transitoire qui correspond à la mise en mouvement du système et éventuellement une partie «vibratoire » qui se développe à partir $\mathrm{du}$ front de chargement.

L'objet de cet article était de présenter l'observation expérimentale des ondes accompagnant le front de chargement par une détonation. Le dispositif permet de mesurer les ondes de déformations avant que celles-ci ne soient influencées par les réflexions par les bords. L'étude s'appuie sur les connaissances établies concernant les détonations, en particulier, sur les résultats applicables à la dynamique du champ de pression réfléchie et à la caractérisation de l'énergie de la charge explosive [6].

L'étude expérimentale met en évidence la corrélation entre les résultats expérimentaux et ceux de l'étude théorique linéaire qui décrit, selon l'intensité et la vitesse de déplacement du chargement, les ondes dans la plaque ou le liquide. Dans la chronologie de la réponse couplée, les ondes accompagnant le front de chargement sont les plus précoces. Si l'amplitude de ces ondes est suffisante, des seuils de transformations (plastification de la plaque ou cavitation dans le liquide par exemple) peuvent être atteints. Cependant, les détonations qui ont été créées (énergie inférieure à un gramme d'équivalent TNT) n'ont pas permis d'observer de réponse non-linéaire accompagnant le front de chargement.

La cohérence théorique est assurée par la double étude analytique et numérique. La pertinence du modèle est établie par l'étude expérimentale. De plus, le modèle linéaire associe la similitude énergétique et mécanique dans un problème d'interaction fluide-structure, permettant ainsi d'étendre les prévisions à différentes échelles, pour une grande variété de chargements mobiles. 


\section{Références}

[1] J. Renard, A. Langlet, O. Pennetier, Response of a large plate-liquid system to a moving pressure step. Transient and stationary aspects, J. Sound and Vibration 265 (2003) 699-724

[2] H. Reismann, Elastic Plates, Theory and Application, Wiley, New York, 1988, 61

[3] A. Lannoy, Analyse des explosions air-hydrocarbures en milieu libre : étude déterministe et probabiliste du scénario d'accident. Prévision des effets de surpression, Bulletin Direction Études et Recherche EDF, 1984

[4] W.E. Baker, P.A. Cox, P.S. Westine, J.J. Kulesz, R.A. Strehlow, Explosion hazards and evaluation, Fundamentals studies in enginerring, Elsevier, 1983, vol. 5
[5] J. Renard, M. Taazount, Transient responses of beams and plates subject to travelling loads. Miscellaneous results, European J. Mechanics A/Solids 21 (2002) $301-322$

[6] J. Brossard, C. Desrosier, H. Purnomo, J. Renard, Pressure loads on plane surface submitted to an explosion, in R. Brun, L.Z. Dumitrescu (ed.), 19th International Symposium on Shock Waves, Springer-Verlag, Berlin, Heidelberg, Université de Provence, Marseille, France, 1993, 4, 387-392

[7] R. Haberman, Elementary applied partial differential equations with Fourier series and boundary value problems, Prentice-Hall, Upper Saddle River, 1998 